\title{
Structure Based Computational Exploration of Beilschmiedia Compounds with Selected Targets against Multidrug-Resistant Mycobacterium tuberculosis
}

\author{
Mohammad Yasir ${ }^{1}$, Pushpendra Singh"1, Sunidhi Chohan², Rahul Shrivastava1,* \\ ${ }^{1}$ Department of Biological Science and Engineering, Maulana Azad National Institute of Technology Bhopal, Madhya Pradesh, INDIA. \\ ${ }^{2}$ Department of Biotechnology, Dr. Hari Singh Gour Central University Sagar, Madhya Pradesh, INDIA.
}

\begin{abstract}
Aim/Background: Tuberculosis is a serious health issue across the world. Various bioactive molecules show the affects against Multidrug-resistant tuberculosis (MDR-TB). There are various standard drugs developed against Mycobacterium for its treatment. But, the pathogen of tuberculosis is developing resistance towards the various standard. The present study was aimed to determine the effect of Beilschmiedia phytoconstituents against different protein targets of Mycobacterium tuberculosis (MTB). Materials and Methods: Proteins and ligands were retrieved from RCSB PDB and Knapsack 3D database. Data of active site of proteins were taken from Protein Data Bank Japan (PDBj) (https:// pdbj.org/) and computed atlas of surface topography of proteins (CASTp) (http://sts. bioe.uic.edu/castp/index.html). Molecular docking was performed by AutoDock tool 4.0. Results: The Beilschmie flavonoid A and Beilschmie flavonoid B, Beilschmiedic Acid A and $B$ were virtually screened for their free binding energy against various protein targets of MTB. Flavonoids showed binding with target proteins and exhibited well promising inhibition of MTB target proteins. There were some specific targets to which particular ligand binds strongly. Conclusion: We found that compounds of Beilschmiedia act in fatty acid degradation pathway at their various steps.
\end{abstract}

Key words: Mycobacterium tuberculosis, Multi-Drug Resistant Tuberculosis, Beilschmiedia zenkeri, Beilschmiedia anacardioides, Molecular Docking.

\section{INTRODUCTION}

Tuberculosis (TB) is a serious health issue across the world. It is an infectious disease caused by the bacillus Mycobacterium tuberculosis (MTB). Disease called "Consumption" in past because the way it would consume from within anyone who became infected. ${ }^{1}$ About 64\% TB patients are from countries like India, Indonesia, China, Philippines, Pakistan, Nigeria and South Africa and India tops the highest number of $\mathrm{TB}$ cases followed by the other six countries respectively. ${ }^{2}$ MTB that causes tuberculosis develops resistance to antimicrobial drugs used to cure disease this condition is known as drug-resistance TB. $^{3}$ There are two main types of drug-resistant TB i.e., Multidrugresistant TB (MDR-TB) and Extensively drug-resistant TB (XDR-TB). Multidrugresistant TB (MDR-TB) is a type of TB that does not respond to at least isoniazid and rifampicin, which is the most powerful anti-TB drugs. ${ }^{4}$ Drug resistance may be because of inadequate treatment or direct transmission of drug-resistant bacillus from one person to another. ${ }^{5}$ The major health problem threatens the progress made in $\mathrm{TB}$ control worldwide. There is an urgent need to develop novel anti-tuberculosis agents, adjunct treatments and to improve immunity. In addition, most of the anti-TB drugs
Submission Date: 06-11-2018; Revision Date: 02-01-2019; Accepted Date: 20-03-2019.

DOI: 10.5530/ijper.53.2s.59 Correspondence: Dr. Rahul Shrivastava Department of Biological Science and Engineering Maulana Azad National Institute of Technology Bhopal, Madhya Pradesh, INDIA.

Phone: +91-9981753702

E-mail: shrivastavarm1972@ gmail.com

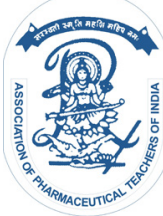

www.ijper.org 
currently target metabolic reactions and proteins that are critical for the proliferation of M. tuberculosis. ${ }^{6}$

In recent years, there has been an increasing interest in natural product based medicines from plant origin. These plants are an important source of bioactive secondary metabolites, which have enormous therapeutic potential. ${ }^{8}$ Therefore, to overcome the serious issue of MDR-TB, plant metabolites can used. In this context, we will study the plant metabolites of two different species of Beilschmiedia i.e., Beilschmiedia anacardioides and Beilschmiedia zenkeri. Beilschmiedia anacardioides and Beilschmiedia zenkeri is an evergreen tree, distributed throughout Central Africa. The fruits of Beilschmiedia zenkeri are appetite stimulants. ${ }^{9}$ Beilschmiedia anacardioides stem bark cure uterine tumours, rubella, female genital infections and rheumatisms. ${ }^{10}$ The stem bark of B. zenkeri consists of four new methoxylated flavonoid derivatives, (2S, 4R)-5, 6, 7-trimethoxyflavan-4-ol, (2S, 4R)-4, 5, 6, 7-tetramethoxyflavan, beilschmie flavonoid $\mathrm{A}$ and beilschmie flavonoid $\mathrm{B}$ and with seven known compounds. They showed antibacterial effect against three strains of bacteria, Pseudomonas agarici, Bacillus subtilis and Streptococcus minor and for their antiplasmodial activity against Plasmodium falciparum, chloroquine-resistant strain $W 2 .{ }^{11}$ Molecular docking is a computer simulation procedure to predict the conformation of a receptor-ligand complex, where the receptor is usually a protein or a nucleic acid molecule (DNA or RNA) and the ligand is either a small molecule or another protein. Objective of current study is to find the potential protein targets of MTB form docking.

\section{MATERIALS AND METHODS}

\section{Retrieval of 3D Structure of Protein and Ligands}

Molecular docking was performed using AutoDock 4.0 software. ${ }^{12}$ Different target sites were selected by getting locus information of organism from target databases; 27 different targeted proteins were selected for docking Table 1. Their 3D structure were retrieved from RCSB PDB in pdb (PDB (TEXT)) file format. ${ }^{13}$ Active sites of proteins were identified from Protein Data Bank Japan (PDBj) (https://pdbj.org//) ${ }^{14}$ and Computed Atlas of Surface Topography of proteins (CASTp) (http://sts. bioe.uic.edu/castp/index.html). ${ }^{15}$ The ligands retrieved from KNApSAcK-3D (http://knapsack3d.sakura. ne.jp/). The Ligand 3-D structures saved in 'mol' file. Files are converted in.pdb format, using Open babel Software version 2.3.2 $\mathrm{a}^{16}$ Table 2, Figure 1.

\section{Protein Ligand Interaction with AutoDock 4.0}

The crystal structure of proteins retrieved from the Protein Data Bank. All polar hydrogen atoms are added and partial charges are placed. Default values of atomic solvation parameters were used throughout the calculations. The grid maps of the protein calculated using the Auto Grid program. Docking simulations done with AutoDock 4.0 using Lamarckian genetic algorithm. The standard docking procedure used for rigid protein and flexible ligand whose torsion angles identified. A grid of 100,100 and 100 points in $\mathrm{x}, \mathrm{y}$ and $\mathrm{z}$ directions was built centered on the catalytic site of the protein. The default settings were used for all other parameters. All calculations were carried out on PC based machines running UBUNTU as operating systems. The results were analyzed using AutoDock Tools.

\section{RESULTS}

\section{Docking of MTB targets with beilschmie flavonoid A}

In silico molecular docking studies was performed using MTB protein targets with beilschmie flavonoid A (B. zenkeri). 13 target proteins shows interaction with beilschmie flavonoid A. All interactions with their binding energy are shown in Table 3. The docked ligands had binding energy ranging between $-0.45 \mathrm{Kcal} / \mathrm{mol}$ to $-2.77 \mathrm{Kcal} / \mathrm{mol}$. The protein Zinc-substituted rubredoxin $\mathrm{B}$ exhibit excellent binding against beilschmie flavonoid A with binding energy $-2.77 \mathrm{Kcal} / \mathrm{mol}$ Figure 2a.

\section{Docking of MTB Targets with beilschmie flavonoid B}

Only 13 target proteins of MTB were docked with the ligand beilschmie flavonoid B. All the interactions with their binding energy are showed in Table 4 . The results showed that all the docked ligands had binding energy ranging between $-0.01 \mathrm{Kcal} / \mathrm{mol}$ to $-3.50 \mathrm{Kcal} / \mathrm{mol}$. The protein enoyl-CoA hydratase echA6 exhibit best binding against beilschmie flavonoid $\mathrm{B}$ with binding energy $-3.50 \mathrm{Kcal} / \mathrm{mol}$ Figure $2 \mathrm{~b}$.

\section{Docking of MTB Targets with Beilschmiedic acid A}

Beilschmiedic acid A and beilschmiedic acid B was obtained from stem bark of B. anacardioides plant. 23 target proteins of MTB interact with beilschmiedic acid. All the interactions with binding energy are shown in Table 5. The results showed that all the docked ligands had binding energy ranging between $-1.71 \mathrm{Kcal} / \mathrm{mol}$ 
Table 1: Selected target proteins of MTB for docking and their function.

\begin{tabular}{|c|c|c|}
\hline Protein Name (PDB-ID) & Classification & Function \\
\hline Antigen $85 \mathrm{C}$ (1dqz) & Immune system & $\begin{array}{l}\text { Facilitates attachment of MTB to murine alveolar } \\
\text { macrophages (AMs). Maintain the integrity of the cell wall. }\end{array}$ \\
\hline $\begin{array}{l}\text { Cytochrome P450 } 14 \text { alpha-sterol demethylase } \\
\text { (CYP51) (1e9x) }\end{array}$ & oxidoreductase & Essential enzyme in sterol biosynthesis in eukaryotes \\
\hline $\begin{array}{c}\text { Crystal structure of 3-bromopyruvate modified } \\
\text { isocitrate lyase (icl) (1f8m) }\end{array}$ & Lyase & $\begin{array}{c}\text { Catalyzes reversible formation of succinate and glyoxylate } \\
\text { a key step of the glyoxylate cycle, which replenish } \\
\text { the tricarboxylic acid cycle during growth on fatty acid } \\
\text { substrates. }\end{array}$ \\
\hline $\begin{array}{l}\text { Mycolic acid cyclopropane synthase } \mathrm{CmaA2} \\
\text { (1 kpi) }\end{array}$ & Transferase & Major components of the cell wall. \\
\hline $\begin{array}{l}\text { Mycolic acid cyclopropane synthase PcaA } \\
\text { (111e) }\end{array}$ & Transferase & $\begin{array}{l}\text { Important for pathogenesis and PcaA catalyze } \\
\text { cyclopropanation at the proximal position of the } \\
\text { meromycolate chain. }\end{array}$ \\
\hline $\begin{array}{l}\text { Peptidyl-prolyl cis-trans isomerase A, PpiA } \\
\text { (1w74) }\end{array}$ & Isomerase & $\begin{array}{l}\text { Important for protein folding and also participate in } \\
\text { signalling, cell surface recognition, chaperoning and heat- } \\
\text { shock response. }\end{array}$ \\
\hline Shikimate kinase (1zyu) & $\begin{array}{l}\text { Signaling protein } \\
\text { transferase }\end{array}$ & $\begin{array}{l}\text { Catalyzes the reaction of phosphoryl transfer from ATP to } \\
\text { shikimic acid (SA). }\end{array}$ \\
\hline Transcriptional regulatory protein EmbR (2ff4) & Transcription & $\begin{array}{c}\text { Positively regulates the transcription of the embCAB operon. } \\
\text { Exhibits ATPase and GTPase activities. }\end{array}$ \\
\hline Hydroxymycolate synthase MmaA4 (2fk7) & Transferase & $\begin{array}{l}\text { Catalyze introduction of methyl branch together with an } \\
\text { adjacent hydroxyl group essential for the formation of both } \\
\text { keto- and methoxymycolates. }\end{array}$ \\
\hline Zinc-substituted rubredoxin B (2kn9) & Electron transport & Play as electron carrier in $\omega$-Hydroxylation of fatty acids. \\
\hline Cell division protein FtsZ $(2 q 1 x)$ & $\begin{array}{l}\text { Cell cycle signaling } \\
\text { protein }\end{array}$ & $\begin{array}{l}\text { Essential cell division protein that forms a contractile ring } \\
\text { structure ( } Z \text { ring) at the future cell division site and The } \\
\text { regulation of the ring assembly controls the timing and the } \\
\text { location of cell division. }\end{array}$ \\
\hline $\begin{array}{l}\text { CFP10 (Culture filtrate protein)-ESAT6 (Early } \\
\text { secreted antigen target) (3fav) }\end{array}$ & Viral protein & $\begin{array}{c}\text { Acts as a strong host (human) T-cell antigen and Involved } \\
\text { in translocation of bacteria from the host (human) } \\
\text { phagolysosome to the host cytoplasm. }\end{array}$ \\
\hline DNA gyrase reaction core (3ig0) & Isomerase & Involved in the regulation of DNA topology. \\
\hline DNA gyrase reaction core $(3 \mathrm{~m} 4 \mathrm{i})$ & Isomerase & Involved in the regulation of DNA topology. \\
\hline Serine/threonine-protein kinase PknB (3ori) & Transferase & PknB, plays a key role in regulating growth and division. \\
\hline $\begin{array}{l}\text { Phosphothreonine-dependent FHA domain } \\
\qquad \text { (3po8) }\end{array}$ & $\begin{array}{l}\text { Peptide binding } \\
\text { protein }\end{array}$ & $\begin{array}{l}\text { FHA domains are well established as phospho-dependent } \\
\text { binding modules mediating signal transduction in Ser/Thr } \\
\text { kinase signaling networks in both eukaryotic and prokaryotic } \\
\text { species. }\end{array}$ \\
\hline $\begin{array}{l}\text { 3-hydroxyacyl-thioester dehydratase } \mathrm{HtdX} \\
\text { (3wew) }\end{array}$ & Lyase & $\begin{array}{c}\text { 3-hydroxyacyl-[acyl-carrier-protein] dehydratase activity, } \\
\text { 3-hydroxyacyl-CoA dehydratase activity and fatty acid } \\
\text { synthase activity. }\end{array}$ \\
\hline $\begin{array}{l}\text { Lipoamide channel-binding sulfonamides } \\
\qquad(4 \mathrm{~m} 52)\end{array}$ & Oxidoreductase & It is a metabolic and detoxifying enzyme. \\
\hline Ribosomal protein S1 (4nni) & Ribosomal protein & $\begin{array}{l}\text { It plays a role in trans-translation; binds tmRNA (the product } \\
\text { of the ssrA gene). }\end{array}$ \\
\hline $\begin{array}{l}\text { 3-hydroxyacyl-thioester dehydratase HtdX } \\
\text { (40ob) }\end{array}$ & Oxidoreductase & $\begin{array}{l}\text { It is involved in synthesis of fatty acid synthase (FAS II), } \\
\text { which is involved in synthesis of mycolic acid present in cell } \\
\text { wall. }\end{array}$ \\
\hline $\begin{array}{l}\mathrm{S} / \mathrm{T} \text { (Serine - Threonine) protein kinase PknG } \\
(4 \mathrm{y} 12)\end{array}$ & Transferase & $\begin{array}{l}\text { A soluble enzyme that controls central metabolism in } \\
\text { Actinobacteria and has been linked to MTB infectivity. }\end{array}$ \\
\hline Lipoarabinomannan carrier protein LprG (4zra) & Lipid binding protein & $\begin{array}{l}\text { Regulate Triacylglyceride Levels, growth rate and virulence } \\
\text { in Mycobacterium tuberculosis. }\end{array}$ \\
\hline enoyl-CoA hydratase echA6 (5duf) & Lipid binding protein & It is essential for $\beta$-oxidation in fatty acid metabolism. \\
\hline Serine/threonine-protein kinase Pknl (5m06) & Signaling protein & $\begin{array}{l}\text { It Plays an important role in slowing down the growth of } \\
\text { mycobacteria within the infected host. }\end{array}$ \\
\hline
\end{tabular}




\begin{tabular}{|c|c|c|}
\hline Table 1: Con' & Transcription & $\begin{array}{c}\text { Involved in the repression of the monooxygenase EthA } \\
\text { which is responsible of the formation of the active metabolite } \\
\text { of ethionamide (ETH). }\end{array}$ \\
\hline Beta>MTBSI of MTB RNA polymerase (5uh7) & Transcription & $\begin{array}{c}\text { DNA-dependent RNA polymerase catalyzes the } \\
\text { transcription of DNA into RNA using the four ribonucleoside } \\
\text { triphosphates as substrates. }\end{array}$ \\
\hline PE family protein PE8 (5xfs) & Protein transport & $\begin{array}{c}\text { It is a family of proteins, which is unique to mycobacteria and } \\
\text { essential for infection, antigenic variation and host-pathogen } \\
\text { interactions. }\end{array}$ \\
\hline
\end{tabular}

\section{Table 2: Selected ligands for docking with their rep- resentative source and molecular formula.}

\begin{tabular}{|c|c|c|}
\hline Name of Metabolite & Source & Molecular formula \\
\hline $\begin{array}{c}\text { Beilschmieflavonoid } \\
\text { A }\end{array}$ & B. zenkeri & $\mathrm{C}_{36} \mathrm{H}_{38} \mathrm{O}_{9}$ \\
\hline $\begin{array}{c}\text { Beilschmieflavonoid } \\
\text { B }\end{array}$ & B. zenkeri & $\mathrm{C}_{35} \mathrm{H}_{36} \mathrm{O}_{9}$ \\
\hline $\begin{array}{c}\text { Beilschmiedic acid A } \\
\text { Beilschmiedic acid } \\
\text { B }\end{array}$ & B. anacardiolides & $\mathrm{C}_{22} \mathrm{H}_{32} \mathrm{O}_{3}$ \\
\hline
\end{tabular}

to $-5.12 \mathrm{Kcal} / \mathrm{mol}$. The protein 3-hydroxyacyl-thioester dehydratase HtdX exhibit good binding against beilschmiedic acid A with binding energy $-5.12 \mathrm{Kcal} /$ mol Figure 2c.

\section{Docking of MTB Targets with beilschmiedic acid B}

17 target proteins of MTB were docked with the ligand beilschmiedic acid B. All interactions with their binding energy are shown in Table 6. The binding energy ranging between $-1.33 \mathrm{Kcal} / \mathrm{mol}$ to $-6.35 \mathrm{Kcal} / \mathrm{mol}$. The protein 3-hydroxyacyl-thioester dehydratase HtdX exhibited highest binding against beilschmiedic acid B with binding energy $-6.35 \mathrm{Kcal} / \mathrm{mol}$ Figure $2 \mathrm{~d}$.

\section{DISCUSSION}

In silico screening methods are extensively used to reduce cost and time of drug discovery. It has been clearly demonstrated that in-silico approach utilized in this study is successful for finding novel natural drugs like 1. Beilschmie flavonoid A, 2. Beilschmie flavonoid B, 3. Beilschmiedic Acid A and 4. Beilschmiedic Acid B which according to literature are present in stem bark of Beilschmiedia zenkeri (1 and 2) and Beilschmiedia anacardioides. These compounds have showed efficient binding with different protein targets of Mycobacterium tuberculosis.

Chen et al. ${ }^{17}$ investigated the in vitro antitubercular effects of compounds isolated from Beilschmiedia plants. In another study Beilschmiedia obscura is reported to fight
Table 3: Docking results of Beilschmieflavonoid A with different Mtb drug target proteins.

\begin{tabular}{|c|c|c|}
\hline Protein Name & $\begin{array}{l}\text { PDB- } \\
\text { ID }\end{array}$ & $\begin{array}{c}\text { Binding } \\
\text { Energy } \\
\text { (Kcal/ } \\
\text { mol) }\end{array}$ \\
\hline Antigen $85 \mathrm{C}$ & $1 \mathrm{dqz}$ & -0.32 \\
\hline $\begin{array}{c}\text { Cytochrome P450 } 14 \text { alpha-sterol } \\
\text { demethylase (CYP51) }\end{array}$ & $1 e 9 x$ & - \\
\hline $\begin{array}{l}\text { Crystal structure of 3-bromopyruvate } \\
\text { modified isocitrate lyase (ICL) }\end{array}$ & $1 f 8 m$ & - \\
\hline Mycolic acid cyclopropane synthase $\mathrm{CmaA} 2$ & $1 \mathrm{kpi}$ & -1.35 \\
\hline Mycolic acid cyclopropane synthase PcaA & $1 / 1 \mathrm{e}$ & -1.29 \\
\hline Peptidyl-prolyl cis-trans isomerase A, PpiA & $1 w 74$ & - \\
\hline Shikimate kinase & $1 z y u$ & -2.54 \\
\hline Transcriptional regulatory protein EmbR & $2 \mathrm{ff} 4$ & -2.32 \\
\hline Hydroxymycolate synthase MmaA4 & $2 \mathrm{fk} 7$ & - \\
\hline Zinc-substituted rubredoxin B & $2 \mathrm{kn} 9$ & -2.77 \\
\hline Cell division protein FtsZ & $2 q 1 x$ & -0.45 \\
\hline $\begin{array}{l}\text { CFP10 (Culture filtrate protein)-ESAT6 } \\
\text { (Early secreted antigen target) }\end{array}$ & 3 fav & - \\
\hline DNA gyrase reaction core & 3ig0 & -2.75 \\
\hline DNA gyrase reaction core & $3 m 4 i$ & - \\
\hline Serine/threonine-protein kinase PknB & 3ori & - \\
\hline Phosphothreonine-dependent FHA domain & 3 po8 & - \\
\hline 3-hydroxyacyl-thioester dehydratase $\mathrm{HtdX}$ & 3 wew & - \\
\hline Lipoamide channel-binding sulfonamides & $4 \mathrm{~m} 52$ & - \\
\hline Ribosomal protein S1 & $4 \mathrm{nni}$ & -1.40 \\
\hline 3-hydroxyacyl-thioester dehydratase $\mathrm{HtdX}$ & $40 o b$ & -2.69 \\
\hline $\begin{array}{c}\mathrm{S} / \mathrm{T} \text { (Serine - Threonine) protein kinase } \\
\text { PknG }\end{array}$ & $4 \mathrm{y} 12$ & -1.56 \\
\hline Lipoarabinomannan carrier protein LprG & $4 z r a$ & -2.49 \\
\hline enoyl-CoA hydratase echA6 & 5 duf & - \\
\hline Serine/threonine-protein kinase Pknl & $5 \mathrm{m06}$ & - \\
\hline HTH-type transcriptional regulator EthR & $5 \mathrm{mxv}$ & - \\
\hline Beta'MtbSI of Mtb RNA polymerase & 5uh7 & -1.42 \\
\hline PE family protein PE8 & $5 \times f s$ & - \\
\hline
\end{tabular}




\begin{tabular}{|c|c|c|}
\hline Protein Name & $\begin{array}{l}\text { PDB- } \\
\text { ID }\end{array}$ & $\begin{array}{l}\text { Binding } \\
\text { Energy } \\
\text { (kcal/mol) }\end{array}$ \\
\hline Antigen 85C & $1 \mathrm{dqz}$ & -3.35 \\
\hline $\begin{array}{c}\text { Cytochrome P450 } 14 \text { alpha-sterol } \\
\text { demethylase (CYP51) }\end{array}$ & $1 e 9 x$ & - \\
\hline $\begin{array}{l}\text { Crystal structure of 3-bromopyruvate } \\
\text { modified isocitrate lyase (ICL) }\end{array}$ & $1 \mathrm{f} 8 \mathrm{~m}$ & - \\
\hline $\begin{array}{l}\text { Mycolic acid cyclopropane synthase } \\
\text { PcaA }\end{array}$ & $1 \mathrm{kpi}$ & -3.01 \\
\hline $\begin{array}{c}\text { Mycolic Acid Cyclopropane } \\
\text { SynthasePcaA }\end{array}$ & $111 \mathrm{e}$ & -1.03 \\
\hline $\begin{array}{c}\text { Peptidyl-prolyl cis-trans isomerase A, } \\
\text { PpiA }\end{array}$ & $1 w 74$ & - \\
\hline Shikimate kinase & $1 z y u$ & -1.93 \\
\hline Transcriptional regulatory protein EmbR & $2 \mathrm{ff} 4$ & -1.29 \\
\hline Hydroxymycolate synthase MmaA4 & $2 \mathrm{fk} 7$ & - \\
\hline Zinc-substituted rubredoxin B & $2 \mathrm{kn} 9$ & -3.09 \\
\hline Cell division protein FtsZ & $2 q 1 x$ & - \\
\hline $\begin{array}{l}\text { CFP10 (Culture filtrate protein)-ESAT6 } \\
\text { (Early secreted antigen target) }\end{array}$ & 3 fav & - \\
\hline DNA gyrase reaction core & $3 i g 0$ & -2.12 \\
\hline DNA gyrase reaction core & $3 m 4 i$ & - \\
\hline Serine/threonine-protein kinase PknB & 3ori & - \\
\hline $\begin{array}{l}\text { Phosphothreonine-dependent FHA } \\
\text { domain }\end{array}$ & 3 po8 & - \\
\hline $\begin{array}{l}\text { 3-hydroxyacyl-thioester dehydratase } \\
\text { HtdX }\end{array}$ & 3wew & -1.33 \\
\hline Lipoamide channel-binding sulfonamides & $4 m 52$ & -1.24 \\
\hline Ribosomal protein S1 & $4 \mathrm{nni}$ & -0.01 \\
\hline $\begin{array}{l}\text { 3-hydroxyacyl-thioester dehydratase } \\
\text { HtdX }\end{array}$ & $400 b$ & - \\
\hline $\begin{array}{c}\mathrm{S} / \mathrm{T} \text { (Serine - Threonine) protein kinase } \\
\text { PknG }\end{array}$ & $4 \mathrm{y} 12$ & - \\
\hline Lipoarabinomannan carrier protein LprG & 4zra & - \\
\hline enoyl-CoA hydratase echA6 & 5duf & -3.50 \\
\hline Serine/threonine-protein kinase Pknl & $5 \mathrm{~m} 06$ & -2.25 \\
\hline HTH-type transcriptional regulator EthR & $5 m \times v$ & - \\
\hline Beta'MtbSI of Mtb RNA polymerase & 5 uh7 & -0.76 \\
\hline PE family protein PE8 & $5 x f s$ & - \\
\hline
\end{tabular}

against infectious diseases caused by gram-negative bacteria and MDR phenotypes. ${ }^{18}$

The conformations of the docked compounds of Beilschmiedia zenkeri and Beilschmiedia anacardioides fits exactly into the active site region of receptor.

To evaluate the mechanism of the compounds with their respective targeted proteins we constructed the predicted pathway for drug-target mechanism Figure 3. We

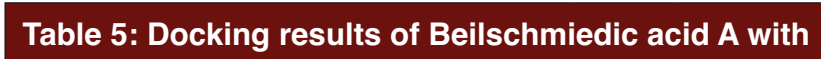
different Mtb drug target proteins.

\begin{tabular}{|c|c|c|}
\hline Protein Name & $\begin{array}{l}\text { PDB- } \\
\text { ID }\end{array}$ & $\begin{array}{c}\text { Binding } \\
\text { Energy } \\
\text { (Kcal/ } \\
\text { mol) }\end{array}$ \\
\hline ANTIGEN 85C & $1 \mathrm{dqz}$ & -4.31 \\
\hline $\begin{array}{c}\text { Cytochrome P450 } 14 \text { alpha-sterol } \\
\text { demethylase (CYP51) }\end{array}$ & $1 e 9 x$ & -2.79 \\
\hline $\begin{array}{l}\text { Crystal structure of 3-bromopyruvate } \\
\text { modified isocitrate lyase (icl) }\end{array}$ & $1 \mathrm{f} 8 \mathrm{~m}$ & - \\
\hline $\begin{array}{l}\text { Mycolic acid cyclopropane synthase } \\
\text { CmaA2 }\end{array}$ & $1 \mathrm{kpi}$ & -2.12 \\
\hline Mycolic Acid Cyclopropane Synthase PcaA & $1 / 1 \mathrm{e}$ & -2.16 \\
\hline Peptidyl-prolyl cis-trans isomerase A, PpiA & $1 w 74$ & -4.62 \\
\hline Shikimate kinase & $1 z y u$ & -2.92 \\
\hline Transcriptional regulatory protein $\mathrm{EmbR}$ & $2 \mathrm{ff} 4$ & -3.76 \\
\hline Hydroxymycolate synthase MmaA4 & $2 \mathrm{fk} 7$ & -2.36 \\
\hline Zinc-substituted rubredoxin B & $2 \mathrm{kn} 9$ & -3.51 \\
\hline Cell division protein FtsZ & $2 q 1 x$ & -2.55 \\
\hline $\begin{array}{l}\text { CFP10 (Culture filtrate protein)-ESAT6 } \\
\text { (Early secreted antigen target) }\end{array}$ & $3 f a v$ & - \\
\hline DNA gyrase reaction core & 3ig0 & -2.84 \\
\hline DNA gyrase reaction core & $3 m 4 i$ & -3.82 \\
\hline Serine/threonine-protein kinase PknB & 3ori & -3.12 \\
\hline Phosphothreonine-dependent FHA domain & $3 p o 8$ & -1.71 \\
\hline 3-hydroxyacyl-thioester dehydratase $\mathrm{HtdX}$ & 3 wew & - \\
\hline Lipoamide channel-binding sulfonamides & $4 \mathrm{~m} 52$ & -4.03 \\
\hline Ribosomal protein S1 & $4 \mathrm{nni}$ & -3.0 \\
\hline 3-hydroxyacyl-thioester dehydratase $\mathrm{HtdX}$ & 4oob & -5.12 \\
\hline $\begin{array}{c}\mathrm{S} / \mathrm{T} \text { (Serine - Threonine) protein kinase } \\
\text { PknG }\end{array}$ & $4 y 12$ & -3.49 \\
\hline Lipoarabinomannan carrier protein LprG & $4 z$ ra & - \\
\hline enoyl-CoA hydratase echA6 & 5 duf & -4.06 \\
\hline Serine/threonine-protein kinase Pknl & $5 \mathrm{m06}$ & -4.52 \\
\hline HTH-type transcriptional regulator EthR & $5 \mathrm{mxv}$ & -2.32 \\
\hline Beta'MtbSI of Mtb RNA polymerase & 5uh7 & -1.91 \\
\hline PE family protein PE8 & $5 \times f s$ & -1.79 \\
\hline
\end{tabular}

found that all the compounds act in fatty acid degradation pathway at their various steps. Beilschmie flavonoid A acts on rubredoxin enzyme, which acts as electron carrier in $\omega$-Hydroxylation of fatty acids, beilschmie flavonoid $B$ acts on echA6 enzyme, involved in $\beta$-oxidation of fatty acid metabolism and beilschmiedic acid $A$ and $B$ acts on synthesis of fatty acid synthase (FAS II), which is involved in synthesis of mycolic acid present in cell 


\begin{tabular}{|c|c|c|}
\hline Protein Name & $\begin{array}{l}\text { PDB- } \\
\text { ID }\end{array}$ & $\begin{array}{l}\text { Binding } \\
\text { Energy } \\
\text { (kcal/mol) }\end{array}$ \\
\hline Antigen $85 \mathrm{C}$ & $1 \mathrm{dqz}$ & -3.34 \\
\hline $\begin{array}{c}\text { Cytochrome P450 } 14 \text { alpha-sterol } \\
\text { demethylase (CYP51) }\end{array}$ & $1 e 9 x$ & - \\
\hline $\begin{array}{l}\text { Crystal structure of 3-bromopyruvate } \\
\text { modified isocitrate lyase (icl) }\end{array}$ & $1 f 8 m$ & -2.09 \\
\hline $\begin{array}{l}\text { Mycolic acid cyclopropane synthase } \\
\text { CmaA2 }\end{array}$ & $1 \mathrm{kpi}$ & -3.26 \\
\hline $\begin{array}{c}\text { Mycolic acid cyclopropane synthase } \\
\text { PcaA }\end{array}$ & $111 \mathrm{e}$ & - \\
\hline $\begin{array}{c}\text { Peptidyl-prolyl cis-trans isomerase A, } \\
\text { PpiA }\end{array}$ & $1 w 74$ & -2.52 \\
\hline Shikimate kinase & $1 z y u$ & -3.65 \\
\hline Transcriptional regulatory protein EmbR & $2 \mathrm{ff} 4$ & -4.38 \\
\hline Hydroxymycolate synthase MmaA4 & $2 \mathrm{fk} 7$ & - \\
\hline Zinc-substituted rubredoxin B & $2 \mathrm{kn} 9$ & -3.46 \\
\hline Cell division protein FtsZ & $2 q 1 x$ & -2.99 \\
\hline $\begin{array}{l}\text { CFP10 (Culture filtrate protein)-ESAT6 } \\
\text { (Early secreted antigen target) }\end{array}$ & $3 f a v$ & -2.37 \\
\hline DNA gyrase reaction core & $3 i g 0$ & -1.45 \\
\hline DNA gyrase reaction core & $3 m 4 i$ & -2.36 \\
\hline Serine/threonine-protein kinase PknB & 3ori & -2.04 \\
\hline $\begin{array}{c}\text { Phosphothreonine-dependent FHA } \\
\text { domain }\end{array}$ & 3 po8 & - \\
\hline $\begin{array}{l}\text { 3-hydroxyacyl-thioester dehydratase } \\
\text { HtdX }\end{array}$ & 3wew & - \\
\hline Lipoamide channel-binding sulfonamides & $4 \mathrm{~m} 52$ & -2.6 \\
\hline Ribosomal protein S1 & $4 \mathrm{nni}$ & -1.33 \\
\hline $\begin{array}{l}\text { 3-hydroxyacyl-thioester dehydratase } \\
\text { HtdX }\end{array}$ & 4oob & -6.35 \\
\hline $\begin{array}{c}\mathrm{S} / \mathrm{T} \text { (Serine }- \text { Threonine) protein kinase } \\
\text { PknG }\end{array}$ & $4 y 12$ & - \\
\hline Lipoarabinomannan carrier protein LprG & 4zra & - \\
\hline enoyl-CoA hydratase echA6 & 5 duf & - \\
\hline Serine/threonine-protein kinase Pknl & $5 \mathrm{~m} 06$ & -4.01 \\
\hline HTH-type transcriptional regulator EthR & $5 m \times v$ & -3.2 \\
\hline Beta'MtbSI of Mtb RNA polymerase & 5 uh7 & - \\
\hline PE family protein PE8 & $5 \times f s$ & - \\
\hline
\end{tabular}

wall of Mycobacterium. Finding suggest that Beilschmiedia species can act as multifaceted antibiotic as cell wall, provide structural integrity, permeability and pathogenicity, both structurally and biosynthetically.

This study indicates the importance of phytoconstituents of Beilschmiedia species i.e., B. zenkeri and B.

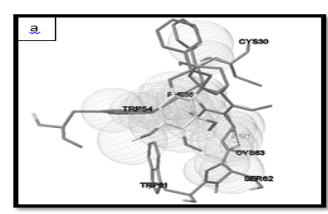

Bcilschmic flavonoid A.

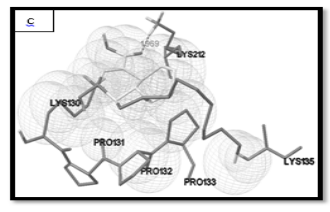

Beilschmiedic acid A

Figure 1: Structure of ligands used in docking
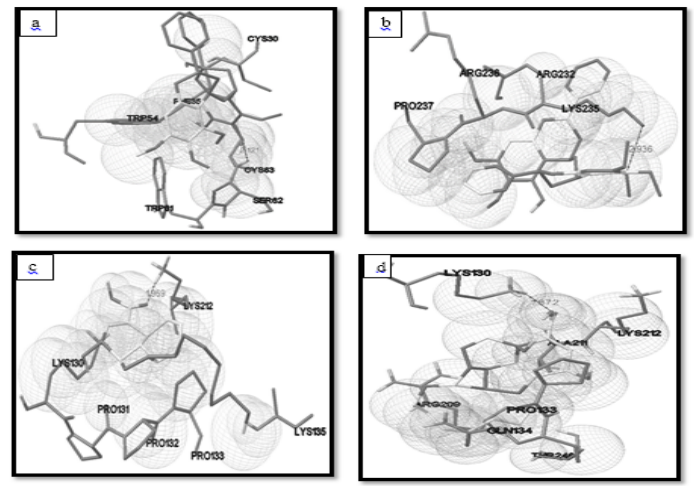

Figure 2: Docking pose of (a) Beilschmie flavonoid A with 2kn9, (b) Beilschmie flavonid B with 5 duf, (c) Beilschmiedic acid A with 40ob, (d) Beilschmiedic acid B with 40ob.

anacardioides and their use as bioactive molecules. The findings suggest that in future these compounds could be developed as a lead compounds for designing of novel drugs against MDR-TB.

\section{CONCLUSION}

Structure based computational exploration have been applied to predict interaction of beilschmie flavonoid A, beilschmie flavonoid B, beilschmiedic acid A and beilschmiedic acid B with MDR-TB targets. Virtual screening strategies are time-saving, cost-effective and productive alternatives in the drug discovery process. In silico molecular docking studies clearly demonstrated binding activity of ligands with protein targets of Mycobacterium tuberculosis which warrants further studies for the development of potent inhibitors in the treatment of MDR-TB. Results clearly indicate that the phytochemicals from Beilschmiedia species as Mycobacterium tuberculosis inhibitors. Hence we conclude that secondary metabolites from Beilschmiedia species could be potential 


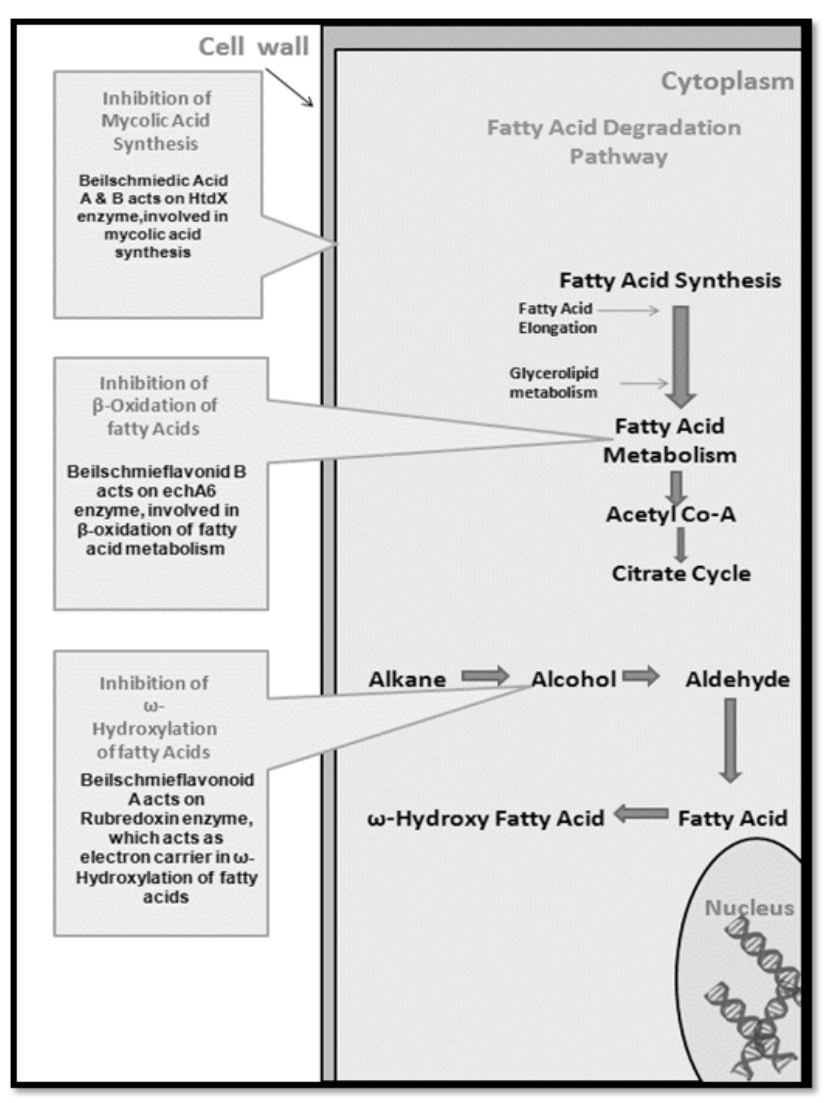

Figure 3: The predicted pathway for drug targets mechanism. Pathway showing that the Beilschmieflavonid $A$ is inhibiting the $\omega$-Hydroxylation of fatty acids; Beilschmieflavonid $B$ is inhibiting $\beta$-oxidation of fatty acids Beilschmiedic Acid $A$ and $B$ is inhibiting mycolic acid synthesis, thus they can be used as drug for MDR-TB.

lead molecules against MDR-TB which can be further evaluated through in vivo studies.

\section{ACKNOWLEDGEMENT}

The authors thank Maulana Azad National Institute of Technology for providing the facilities required for carrying out research work.

\section{CONFLICT OF INTEREST}

The authors declare no conflict of interest.

\section{ABBREVIATIONS}

CASTp: Computed Atlas of Surface Topography of Proteins; DNA: Deoxyribonucleic acid; MTB: Mycobacterium tuberculosis; MDR-TB: Multidrug-Resistant Tuberculosis; PDBj: Protein Data Bank Japan; RNA: Ribonucleic acid; TB: Tuberculosis; XDR-TB: Extensively drug-resistant TB.

\section{REFERENCES}

1. Manchester K. Tuberculosis and leprosy in antiquity: an interpretation. Medical History. 1984;28(2):162-73.

2. Tuberculosis | NIH: National Institute of Allergy and Infectious Diseases [Internet]. Niaid.nih.gov. 2018 [cited 5 November 2018]. Available from: https://www.niaid.nih.gov/diseases-conditions/tuberculosis-tb.

3. Berghuis A, Matlashewski G, Wainberg M, Sheppard D. Handbook of Antimicrobial Resistance. Springer New York. 2017.

4. Treatment of drug-resistant TB: Resources [Internet]. World Health Organization. 2018 [cited 5 November 2018]. Available from: http://www.who. int/tb/areas-of-work/drug-resistant-tb/treatment/resources/en/.

5. Lange C, Abubakar I, Alffenaar J, Bothamley G, Caminero J, Carvalho A, et al. Management of patients with multidrug-resistant/extensively drugresistant tuberculosis in Europe: a TBNET consensus statement. European Respiratory Journal. 2014;44(1):23-63.

6. Adhvaryu M, Vakharia B. Drug-resistant tuberculosis: emerging treatment options. Clinical Pharmacology: Advances and Applications. 2011;3:51.

7. Fabricant $\mathrm{D}$, Farnsworth $\mathrm{N}$. The value of plants used in traditional medicine for drug discovery. Environmental Health Perspectives. 2001;109(suppl 1):69-75.

8. Barbosa RW, Do Nascimento M, Nascimento PL, Costa MF, Ataide SA, Silva JJ, et al. Selecting Medicinal Plants for Development of Phytomedicine and Use in Primary Health Care. Bioactive Compounds in Phytomedicine. 2012.

9. Iwu M. Handbook of African medicinal plants. Boca Raton, Fla: CRC Press. Taylor and Francis Group. 2014.

10. Lenta B, Chouna J, Nkeng-Efouet P, Sewald N. Endiandric Acid Derivatives and Other Constituents of Plants from the Genera Beilschmiedia and Endiandra (Lauraceous). Biomolecules. 2015;5(2):910-42.

11. Lenta B, Tantangmo F, Devkota K, Wansi J, Chouna J, Soh R, et al. Bioactive Constituents of the Stem Bark of Beilschmiedia zenkeri. Journal of Natural Products. 2009;72(12):2130-4.

12. Huey R, Morris G, Olson A, Goodsell D. A semiempirical free energy force field with charge-based desolvation. Journal of Computational Chemistry. 2007;28(6):1145-52.

13. Berman HM, Westbrook J, Feng Z, Gilliland G, Bhat TN, Weissig H, et al. The Protein Data Bank. Nucleic Acids Research. 2000;28:235-42

14. Kinjo A, Bekker G, Suzuki H, Tsuchiya $\mathrm{Y}$, Kawabata T, Ikegawa $\mathrm{Y}$, et al. Protein Data Bank Japan (PDBj): updated user interfaces, resource description framework, analysis tools for large structures. Nucleic Acids Research. 2016;45(D1):D282-8.

15. Binkowski TA, Naghibzadeh S, Liang J. CASTp: Computed Atlas of Surface Topography of proteins. Nucleic Acids Res. 2003;31(13):3352-5.

16. O’Boyle N, Banck M, James C, Morley C, Vandermeersch T, Hutchison G. Open Babel: An open chemical toolbox. Journal of Cheminformatics. 2011;3(1):33.

17. Chen JJ, Chou ET, Peng CF, Chen IS, Yang SZ, Huang HY. Novel epoxyfuranoid lignans and antitubercular constituents from the leaves of Beilschmiedia tsangii. Planta Med. 2007;73(06):567-71.

18. Fankam A, Kuiate J, Kuete V. Antibacterial activities of Beilschmiedia obscura and six other Cameroonian medicinal plants against multi-drug resistant Gram-negative phenotypes. BMC Complementary and Alternative Medicine. 2014;14(1):241. 


\section{PICTORIAL ABSTRACT}

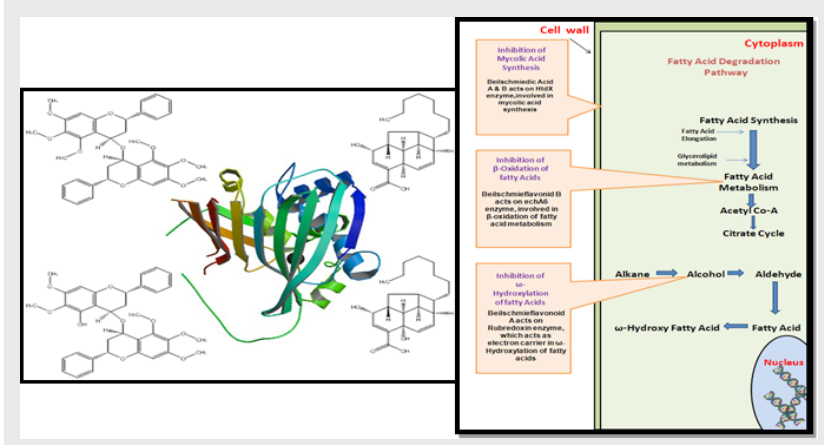

\section{SUMMARY}

- There is a continuous need for the development of new drug molecules with newer targets and with an alternative mechanism of action due to increasing drug resistance of Mycobacterium tuberculosis to the currently used drugs. This study indicates the importance of phytoconstituents of Beilschmiedia species i.e., B. zenkeri and B. anacardioides and their use as bioactive molecules. The findings suggest that in future these compounds could be developed as a lead compounds for designing of novel drugs against MDR-TB.

Cite this article: Yasir M, Singh P, Chohan S, Shrivastava R. Structure Based Computational Exploration of Beilschmiedia Compounds with Selected Targets against Multidrug-resistant Mycobacterium tuberculosis. Indian J of Pharmaceutical Education and Research. 2019;53(2S):s143-s150. 